Muschalla, B., Angerer, P., \& Knaevelsrud, C. (2017). Arbeitsfähigkeitsbeschreibung bei psychischen Erkrankungen. Verhaltenstherapie, 27, 27-34.

\title{
Arbeitsfähigkeitsbeschreibung bei psychischen Erkrankungen
}

\author{
Beate Muschalla $^{1}$, Peter Angerer ${ }^{2}$, Christine Knaevelsrud $^{1}$ \\ ${ }^{1}$ Freie Universität Berlin \\ ${ }^{2}$ Universität Düsseldorf
}

Manuskript zur Publikation in Verhaltenstherapie

45.424 Zeichen (inklusive Abstract und Literatur)

Korrespondenzadresse:

Dr. Beate Muschalla, Freie Universität Berlin, Abteilung Klinische Psychologie und

Psychotherapie, Habelschwerdter Allee 45, 14195 Berlin, Germany, Email:

beate.muschalla@gmx.de 


\title{
Describing work ability due to mental disorders
}

\begin{abstract}
Work ability is a complex phenomenon with increasing importance in the field of mental disorders. In the recent years, increasing rates of disability pensions and sickness absence due to mental disorders have been widely reported. Also therapeutic practitioners are asked to assess and describe work ability (limitations) in a way that allows to develop individually suitable means for restoring work ability or finding work adjustment means. Description of symptoms alone has been found to be not sufficient for work-oriented outcomes. The bio-psycho-social health model of the International Classification of Functioning Disability and Health ICF [WHO, 2001] has offered a level of describing health problems in terms of capacities and activities (complementary to and beyond the level of symptoms only), and a description of impairment severity in dependence of context factors. This review gives an overview on the spectrum of operationalizations of work ability. Referring to the activity/capacity and context idea of the ICF concept, examples for currently available measures for work ability will be described.
\end{abstract}

Keywords: work ability, work performance, work limitations, work capacity, mental disorders, disability, rehabilitation 


\section{Zusammenfassung}

Arbeitsfähigkeit ist ein Thema von zunehmender Bedeutung bei der Behandlung psychischer Erkrankungen. Arbeits- und Erwerbsunfähigkeit aufgrund psychischer Erkrankungen haben in den letzten Jahren (relativ zu anderen Erkrankungsgruppen) zugenommen. Dies bringt in ambulanten wie stationären verhaltenstherapeutischen Behandlungen die Notwendigkeit mit sich, sich intensiver dem Thema der Arbeitsfähigkeit zu widmen. In der Praxis wie auch in der Klinik stellt sich Ärzten und Psychotherapeuten dabei unter anderem die Frage, wie man Arbeitsfähigkeit im Therapieverlauf beschreiben kann. Hier geht es neben der klinischen Diagnostik ggf. auch um die Auswahl von Instrumenten für eine ergänzende standardisierte Verlaufsdokumentation.

Das Krankheitsmodell der Internationalen Klassifikation der Funktionsfähigkeit, Behinderung und Gesundheit ICF [WHO, 2001] bietet mit seinem biopsychosozialen Ansatz eine sinnvolle Ordnungsmöglichkeit von Diagnostik und Therapiezielen. Danach können Diagnostik und Behandlung auf der Symptomebene, auf der Aktivitäts- und Fähigkeitsebene, und auf der Kontextebene unterschieden werden. Die vorliegende Übersicht soll dazu anregen, Arbeitsfähigkeits-Diagnostik vor dem Hintergrund dieser verschiedenen Ebenen zu reflektieren. Sie bietet außerdem Beispiele verschiedener wissenschaftlicher Operationalisierungsvarianten von „Arbeitsfähigkeit“.

Schlüsselwörter: Arbeitsfähigkeit, psychische Erkrankungen, Teilhabe, Rehabilitation 


\section{Einleitung}

Psychische Erkrankungen können am Arbeitsplatz mit „Krankschreibung“ (Arbeitsunfähigkeit) [Lidwall, 2015] oder mit Präsentismus [Esposito et al., 2007] einhergehen. Präsentismus bedeutet eine krankheitsbedingt reduzierte Arbeitsproduktivität. Chronische psychische Beeinträchtigungen können auch zur teilweisen oder vollständigen Erwerbsminderung führen. Bei den hohen Raten von Arbeitsunfähigkeit und Erwerbsminderungsberentung aufgrund psychischer Erkrankungen sind Psychotherapeuten - Psychologen ebenso wie Ärzte zunehmend aufgefordert, auch die Wiederherstellung der Arbeitsfähigkeit ihrer Patienten in ihrer Behandlung zu befördern [BPtK, 2015]. Für den Praktiker wie den Wissenschaftler stellt sich in diesem Zusammenhang die Frage, welche verschiedenen Beschreibungsmöglichkeiten von Arbeitsfähigkeit es gibt.

Diese Übersicht verfolgt zwei Ziele: zum einen soll für die Unterscheidung von sozialmedizinisch/sozialrechtlichem Verständnis von Arbeitsfähigkeit einerseits und wissenschaftlichem Arbeitsfähigkeitsverständnis andererseits sensibilisiert werden. Darauf aufbauend werden Beispiele unterschiedlicher wissenschaftlicher Operationalisierungen gegeben. Diese werden hinsichtlich ihrer Nähe zum sozialmedizinischen Arbeitsfähigkeitsverständnis und hinsichtlich praktischer Aspekte diskutiert. Die konzeptuelle Einordnung mag Entscheidungen erleichtern, für welchen Zweck man welche Art von Arbeitsfähigkeitsbeschreibung verwenden kann. Alle beschriebenen Instrumente liegen in deutscher Sprache vor und wurden in empirischen Studien psychometrisch evaluiert. Sie können in der Praxis die klinischen Diagnostik ergänzen im Sinne einer strukturierten Verlaufsbeschreibung, oder als Explorationshilfen nützen. 


\section{Definitionen von Arbeitsfähigkeit bei psychischen Erkrankungen}

Um die vielfältige Literatur zum Thema Arbeitsfähigkeit zu verstehen, ist es notwendig, ein sozialrechtlich/sozialmedizinisches und ein wissenschaftliches Verständnis von Arbeitsfähigkeit zu unterscheiden.

\section{Sozialrechtlich/sozialmedizinisches Verständnis von Arbeitsfähigkeit}

Sozialrechtlich/sozialmedizinisch gibt es in Deutschland die Begriffe der Arbeitsfähigkeit und der Erwerbsfähigkeit.

Arbeitsunfähigkeit - im Volksmund oft „Krankschreibung“ genannt wird i.d.R. vom niedergelassenen Arzt attestiert. Sie bescheinigt, dass jemand aktuell krankheitsbedingt seine derzeitige berufliche Tätigkeit nicht ausüben kann. Ist jemand arbeitslos, dann bedeutet arbeitsunfähig, dass er/sie krankheitsbedingt aktuell nicht auf dem allgemeinen Arbeitsmarkt tätig werden kann (z.B. keinen Arbeitsamtstermin wahrnehmen oder sich nicht bewerben kann). Eine Arbeitsunfähigkeitsfeststellung kann nicht aufgrund einer Erkrankungssymptomatik allein erfolgen. Sie muss mit Hinblick darauf erfolgen, ob die derzeit ausgeübte Tätigkeit krankheitsbedingt nicht, oder nur mit der Gefahr die Krankheit zu verschlimmern ausgeübt werden kann [GBA, 2014; Foster und Pinals, 2014; Linden et al., 2015]. Dazu ist es notwendig zu wissen, welche Anforderungen der Arbeitsplatz stellt, spezifische Charakteristika der Krankheit zu kennen und wie die krankheitsbedingten Beeinträchtigungen aussehen. Zum Beispiel wäre ein Verkäufer mit einer depressiven Episode arbeitsunfähig, wenn die Depression seine Fähigkeit Verkaufsgespräche zu führen beeinträchtigt. 
Erwerbsfähigkeit meint die überdauernde Leistungsfähigkeit, d.h. ob jemand in sechs Monaten oder darüber hinaus seiner beruflichen Tätigkeit oder einer anderen Tätigkeit auf dem allgemeinen Arbeitsmarkt nachgehen kann. Zu Beispiel könnte ein Patient mit einer austherapierten chronischen sozialen Phobie und der andauernden Unfähigkeit Verkaufsgespräche zu führen zur Ausübung von Verkäufertätigkeiten nicht mehr in der Lage sein. In diesem Zusammenhang könnte es um die Frage gehen, ob für einen Patienten eine Leistung zur Teilhabe am Arbeitsleben [SGB IX § 33] in Frage kommt, z.B. Umsetzung in einen Onlineverkauf, Umschulung oder Vermittlung in ein Tätigkeitsfeld ohne Interaktionsanforderungen. Eine Berentung wegen voller Erwerbsminderung [SGB VI § 43] käme jedoch nur dann in Frage, wenn die Erkrankung an der Ausübung jedweder möglicher Tätigkeit im Umfang von mindestens drei Stunden pro Tag auf dem allgemeinen Arbeitsmarkt hindern würde.

In den verschiedenen Sozialsystemen in Europa wird Arbeitsfähigkeit sozialrechtlich/sozialmedizinisch entweder beschrieben als prozentuale Angabe einer Leistungsfähigkeit, oder in möglichen Arbeitsstunden pro Tag, oder es wird eine Beeinträchtigung im Sinne eines Behinderungsgrades angegeben [Anner et al., 2012].

\section{Entwicklung des wissenschaftlichen Arbeitsfähigkeitsverständnisses}

Arbeitsfähigkeit als wissenschaftlicher Begriff umfasst je nach Definition verschiedene subjektive und objektive Aspekte von Verhalten und Erleben in Bezug auf den Arbeitsplatz oder das Arbeitsleben. Dieses Verständnis von Arbeitsfähigkeit ist nicht zu verwechseln mit der sozialrechtlich/sozialmedizinischen Definition von Arbeitsfähigkeit. Es gibt eine 
breite Palette von wissenschaftlichen Ansätzen zur Beschreibung von Arbeitsfähigkeit, aus der im Folgenden einige Beispiele näher beschrieben werden.

Arbeitsfähigkeit ist in der bisherigen wissenschaftlichen Literatur mit unterschiedlich detailliert und auch inhaltlich heterogen definiert worden [MacDonald et al., 2001; Lederer et al., 2014; Anner et al., 2012]. Dies ist u.a. darauf zurückzuführen, dass Arbeitsfähigkeit in unterschiedlichsten Kontexten untersucht worden ist, z.B. bei speziellen Erkrankungen, oder ausgewählten Berufsgruppen.

In der empirischen Forschung wurde reduzierte Arbeitsfähigkeit lange Zeit als eine unmittelbare Folge von Erkrankung bzw. Symptomen beschrieben [MacDonald-Wilson et al., 2001]. Gleichzeitig zeigte sich jedoch, dass das Symptom an sich - bspw. eine depressive Verstimmung oder eine sorgenvolle Art des Denkens - für Arbeitsfähigkeit keinen Erklärungswert besitzt [Gatchel et al., 1994]. Auch fand sich nur ein geringer Zusammenhang zwischen Symptomverbesserung und Rückkehr zur Arbeit [Cornelius et al., 2011].

Als Reaktion auf die Erkenntnis, dass Symptome keine prognostische Wertigkeit für berufsbezogene Fähigkeiten und Aktivitäten besitzen, wurde dazu übergegangen andere Beschreibungsebenen zu erkunden [MacDonald-Wilson et al., 2001]. Arbeitsfähigkeit ist vom Grundsatz her ein relatives Konzept: Arbeitsfähigkeit ist nicht der Person innewohnend, sondern entsteht aus der Wechselwirkung von Person, Erkrankung, und Arbeitsanforderungen [Lederer et al., 2014]. Auch mit der Einführung der Internationalen Klassifikation der Funktionsfähigkeit, Behinderung und Gesundheit, ICF [WHO, 2001] wurde eine Beschreibungsweise von Gesundheitsproblemen als Beeinträchtigungen in 
verschiedenen Lebensbereichen populär. Über die Symptom-Ebene (z.B. Panikattacke) hinaus können Aktivitätsprobleme (z.B. jemand kann sich nicht unbegleitet frei bewegen) und Teilhabebeeinträchtigungen (z.B. Betroffener kann nicht allein mit dem Auto zur Arbeit fahren) beschrieben werden. Eine Einschränkung der Arbeitsfähigkeit kann in diesem Sinne als erkrankungsbedingte Aktivitäts- und Teilhabebeeinträchtigung beschrieben werden, die nur unter bestimmten Anforderungen zutage tritt [Linden et al., 2015; Anner et al., 2012].

Ein Argument für die Begründung von Arbeitsunfähigkeit über Aktivitätsund Teilhabebeeinträchtigungen ist auch, dass psychische Erkrankungen chronisch sind und Probleme über die Lebensspanne hinweg auftreten [Stansfeld et al., 2008]. Aktivitätsprobleme sind abhängig davon, in welchem Kontext die Symptome stören und in welchem ggf. auch nicht. Oftmals kann nicht die vollständige Heilung der Erkrankung und Entfernung der Symptome das Behandlungsziel sein. Vielmehr geht es darum einen Weg zu finden gut durch das (Arbeits-)Leben zu kommen, auch mit bestehenden oder wiederkehrenden Symptomen. Verhaltenstherapeutische Behandlungsansätze sind zu großem Teil fähigkeits- und aktivitätsorientiert [Muschalla, 2014], wie bspw. soziale Kompetenztrainings, Aktivitätsaufbau, Zeitmanagement und das Erlernen von Problemlösestrategien. Auch wenn bei einer Wiedereingliederung am Arbeitsplatz nach längerer Abwesenheit eine Arbeitsplatzanpassung notwendig wird, geschieht dies fähigkeits- und aktivitätsorientiert: Die Person-Job-Passung [French, 1973] wird dadurch hergestellt, dass Arbeitsanforderungen an das reduzierte Aktivitäts- und Fähigkeitsniveau der Person angepasst werden. Bei einer Neurasthenie und dadurch beeinträchtigter Durchhaltefähigkeit kann bspw. 
eine Verkürzung von Fahrtwegen hilfreich sein um die Arbeitsfähigkeit zu erhalten oder eine Flexibilisierung von Pausenzeiten.

Im Folgenden werden beispielhaft einige gängige Ansätze zur Arbeitsfähigkeitsbeschreibung skizziert und im Hinblick auf ihren Aktivitäts- und Teilhabegehalt diskutiert.

\section{Messung von (Arbeits-)Fähigkeit bei psychischen Erkrankungen}

Es gibt viele verschiedene empirische Ansätze zur Erhebung von Arbeitsfähigkeit(sbeeinträchtigung) [MacDonald-Wilson et al., 2001; Lederer et al., 2014], wie zum Beispiel Globalmaße, quantitative Maße von Abwesenheitszeiten oder Produktivitätsverlust, oder auch differenziertere Maße, die aktivitätsbezogen das Arbeitsverhalten beschreiben. Im Folgenden sollen drei Erhebungsansätze unterschieden werden:

a) Maße für globales Fähigkeits- und Teilhabeniveau, einschließlich Arbeitsfähigkeit,

b) Maße für Arbeitsfähigkeit als Globalkonstrukt

c) Maße für differenzierte Dimensionen von Arbeitsfähigkeit und aktivitäten.

Die im Folgenden beschriebenen Instrumente sind vor allem in den letzten fünf Jahren in Studien mit Menschen mit psychischen Erkrankungen verwendet worden und in deutscher Sprache erhältlich. Die heterogenen Begrifflichkeiten zeigen sich auch bei den Operationalisierungen. Es wird u.a. von „Arbeitsfähigkeit“, “Beeinträchtigung”, "Behinderung”, oder "Performanz” gesprochen. Im Sinne einer übersichtlichen Lesbarkeit werden auch im Folgenden 
die Begrifflichkeiten „Arbeitsfähigkeit“ bzw. „Arbeitsunfähigkeit“" verwendet, im Sinne eines wissenschaftlichen Verständnisses von Arbeitsfähigkeit. Sofern der Bezug zur sozialrechtlich/sozialmedizinischen Arbeitsfähigkeit im engeren Sinne hergestellt wird, wird dies explizit erwähnt.

\section{Beschreibung allgemeiner Beeinträchtigung einschließlich}

\section{Arbeitsfähigkeitsbeeinträchtigung}

Ein bekanntes Beispiel zur Beschreibung der generellen Beeinträchtigung des sozialen Lebens einer Person ist die Global Assessment of Functioning Scale GAF [Sass et al., 1998]. Allerdings kombiniert der GAF Score Symptome (Affekt, formale Denkstörungen, Panikattacken), Fähigkeits- und Aktivitätsprobleme (Konflikte mit Kollegen) und Umweltfaktoren (geringe Anzahl an Bezugspersonen) miteinander. Anhand des globalen GAF Scores konnte daher keine Aussage zur Beeinträchtigung des Fähigkeits- bzw. Aktivitätsniveaus gemacht werden. Der GAF Score wurde in der DSM-5-Version gestrichen und stattdessen das differenziertere WHODAS 2.0 zur Verwendung vorgeschlagen [WHO, 2004].

\section{Beschreibung allgemeiner Arbeitsfähigkeit mittels Globalbeurteilung}

Es gibt verschiedene Globalmaße von Arbeitsfähigkeit: Es wurden beispielsweise allgemeine Maße für Arbeitsleistung verwendet, wie etwa eine Einschätzung der Performanz bei der Arbeit von "niedriger" bis zu "hoher Performanz" [z.B. Tziner et al., 2002]. Hierzu wurde Arbeitsfähigkeit auf einem sehr allgemeinen Niveau von Aktivität beschrieben; es wurde kein direkter Bezug zu Erkrankungen hergestellt. Im Gegensatz dazu gibt es auch Ansätze, die Beeinträchtigungen auf 
ganz bestimmte Ursachen zurückführen, z.B. Ressourcenverlust aufgrund der Arbeitsumgebung [Vinokur et al., 2011]. Dies ist eine stimulus-orientierte Herangehensweise. Man findet solche Stimulus-Orientierung auch häufig in den Krankheitsmodellen von Patienten mit Arbeitsängsten [Muschalla et al., 2014]. Diese Patienten neigen häufig dazu Gesundheitsprobleme external zu attribuieren. Das Ausmaß, in dem Gesundheitsbeschwerden auf Arbeitsbedingungen zurückgeführt werden, kann ein Indiz sein für die Art und das Ausmaß der Psychopathologie. Zunächst nach „Arbeitsproblemen“ zu fragen kann ein Türöffner und Ansatzpunkt sein, mit dem Patienten in der weiteren Exploration auch über arbeitsbezogene Beeinträchtigungen und Ängste zu sprechen [Muschalla et al., 2014]. Externalisierende Krankheitsmodelle, die Patienten äußern, sollten vom Behandler nicht unhinterfragt übernommen oder bestätigt werden, da sie dysfunktionale Verhaltensimpulse befördern können. Auf Aussagen wie „Die Arbeit hat mich krank gemacht! Ob ich nicht lieber kündige...?" sollte therapeutischerseits relativierend und hinterfragend reagiert werden [Muschalla et al., 2014].

Dem sozialrechtlich/sozialmedizinischen Verständnis von Arbeitsfähigkeit [AU-Richtlinien, GBA, 2014] näher kommen Operationalisierungen, die explizit nach krankheitsbedingten Aktivitätsbeeinträchtigungen fragen. Ein Beispiel hierfür ist der Index zur Messung von Beeinträchtigungen zur Teilhabe, IMET [Deck et al., 2007, 2015]. Der IMET ist ein Selbstbeurteilungsfragebogen mit neun Items, der das subjektiv erlebte Ausmaß von Aktivitätsbeeinträchtigung in verschiedenen Lebensbereichen erfasst [Deck et al., 2007; Deck et al., 2015]. In einigen Untersuchungen wurde im IMET ein Item ergänzt, welches die erlebte 
krankheitsbedingte Beeinträchtigung im Bereich “Arbeit und Beruf” erfasst [Muschalla et al., 2012]. Dies kann als ein Globalmaß von subjektiver Arbeitsfähigkeitsbeeinträchtigung verstanden werden.

Ein weltweit genutztes Selbstbeurteilungsverfahren zur Beschreibung von krankheitsbedingter Beeinträchtigung ist die Sheehan Disability Scale [SDS, Sheehan, 1983]. Eines der drei Items bezieht sich auf den Lebensbereich Arbeit. Der Proband wird gefragt, ob die Symptome die Arbeit stören. „Arbeit“"kann hierbei bezahlte, unbezahlte freiwillige Arbeit oder Ausbildung bedeuten. Die SDS wurde in 21 Sprachen übersetzt.

Der Work Ability Index [WAI, Tuomi et al. 1998] ist ein international wissenschaftlich wie auch in der betriebsärztlichen Praxis verwendetes Arbeitsfähigkeits-Maß. Es wird ein Globalindex von Arbeitsfähigkeit gebildet, auf Grundlage verschiedener Einzelinformationen, die in einem fünfzehnminütigen klinischen Interview erhoben werden können. “Arbeitsfähigkeit” im Sinne des WAI ist auf konzeptueller Grundlage des „Haus der Arbeitsfähigkeit" von Ilmarinen entstanden und wird hier verstanden als ein Ergebnis der Wechselwirkung von Person und Umwelt [Ilmarinen und Lehtinen, 2004]. Zunächst wird vom Probanden eine Einschätzung seiner Arbeitsfähigkeit insgesamt erfragt, im zweiten Schritt wird unterschieden nach körperlicher und psychischer Arbeitsfähigkeit. Zusätzlich soll eine Prognose der Arbeitsfähigkeit in zwei Jahren gegeben werden. Weiterhin werden die Anzahl und Art der Erkrankungen erhoben, die Art der erlebten Beeinträchtigung in der Arbeitstätigkeit aufgrund der Erkrankungen (Wohlbefindens-Beeinträchtigung, Notwendigkeit veränderter Arbeitsmethoden, reduzierter Arbeitszeitumfang), sowie eine kurze Globaleinschätzung, ob die Person sich aktiv, hoffnungsvoll und 
froh erlebte in täglichen Routineaktivitäten. Über diese verschiedenen Aspekte wird ein Gesamtindex berechnet. Somit stellt der Work Ability Index einen Globalscore aus sehr verschiedenen Aspekten von Arbeitsfähigkeit und der Erkrankungen selbst dar. In den letzten Jahren wurde der Work Ability Index in deskriptiven Studien mit verschiedenen Berufsgruppen sowie in nationalen bevölkerungsrepräsentativen Stichproben, in klinischen Stichproben und Therapiestudien eingesetzt.

Als Fremdbeurteilungsverfahren ist das Groningen Social Disability Schedule erwähnenswert, in dem die gesundheitsbedingte Beeinträchtigung auf eher globalem Niveau beschrieben wird [GSDS, Wiersma et al., 1988, 1990]. Das GSDS ist ein halbstrukturiertes Interview für soziale Fähigkeitsbeeinträchtigungen. Es basiert auf der sozialen Rollentheorie und umfasst acht soziale Rollen; eine davon ist die Berufsrolle. Das gesamte Interview kann nach einem Interviewertraining durchgeführt werden und dauert ca. eine Stunde. Beeinträchtigungen in sozialen Rollen zeigen sich zunächst in öffentlichen Bereichen und erst bei stärkerer Ausprägung im Bereich von Familie, Freizeit und Freundeskreis [de Jong et al., 1985]. Viele PsychotherapiePatienten beklagen Probleme in zwischenmenschlichen Beziehungen. Auch wenn die Patienten möglicherweise nur ihre Beziehungsprobleme mit Freunden oder Familie berichten, so kann doch die Frage gestellt werden, ob sie ebenso oder erst recht am Arbeitsplatz Probleme mit Kollegen oder Vorgesetzten haben. Es ist also lohnend danach zu fragen, selbst wenn Patienten es nicht von sich aus berichten. Das GSDS beschreibt Beeinträchtigung der Teilhabe und erfordert im arbeitsbezogenen Frageteil Bezugnahme auf einen umschriebenen Kontext. Beeinträchtigung wird dabei als abweichendes Verhalten einer Person gegenüber 
den Regeln der sozialen Umwelt, d.h. gegenüber der normativen Gruppe verstanden. Im GSDS Interview wird der Lebensbereich Arbeit anamnestisch exploriert: Zunächst wird nach Abwesenheitszeiten und -gründen gefragt, ob es Probleme gegeben hat bei der Erfüllung von Rollen im Job und welche Konsequenzen dies hatte. Als Nächstes wird die Arbeitsleistung exploriert. Auch nach der Fremdwahrnehmung wird gefragt, also wie andere die Arbeitsleistung des Patienten wahrnehmen, ob es unzufriedene Rückmeldungen gegeben hat, berechtigte Kritik geäußert wurde, oder es negative Konsequenzen für Minderleistungen gegeben hatte. Ein dritter Frageblock zielt auf die Kontakte mit anderen: Von welcher Qualität ist die Beziehung zu Kollegen, die Arbeitsatmosphäre, gab es Konflikte oder Missverständnisse mit Kollegen, gibt es Kollegen, die der Befragte vermeidet? Für jeden Bereich kann eine Beeinträchtigungsschwere eingeschätzt werden. Die Beurteilungsregeln geben vor, dass mit zunehmender Häufigkeit, Dauer, und Schwere (Selbst- oder Fremdschädigungspotential) der Normabweichung die Beeinträchtigung stärker wird. Die Notwendigkeit der Unterstützung durch andere soll als zusätzliches Einschätzungskriterium mitbedacht werden.

Das World Health Organization Disability Assessment Schedule II (WHODAS 2.0) ist ein erkrankungsübergreifendes Maß für die Erfassung funktionaler Gesundheit und Beeinträchtigung [WHO, 2004]. Mit dem WHODAS 2.0 kann ein weiteres Spektrum gesundheitsassoziierter Aktivitätsund Partizipationsstörungen in den letzten 30 Tagen beschrieben werden. Das WHODAS ist als Selbst- oder Fremdbeurteilungsinstrument verfügbar, mit 12 oder 36 Items. Das WHODAS ist ein validiertes Instrument, das eng angelehnt ist an die ICF und interkulturell eingesetzt werden kann. Das WHODAS möchte 
Aktivitäts- und Teilhabebeeinträchtigungen erfassen, es enthält jedoch auch symptombezogene Items, wie zum Beispiel zur Konzentrationsfähigkeit. In arbeitsbezogenen Items geht hier um Schwierigkeiten, die alltägliche Arbeit in Umfang, Tempo und Qualität zu bewältigen. Die Items differenzieren jedoch nicht zwischen psychologischen Fähigkeiten, die ggf. in der einen oder anderen Berufstätigkeit in unterschiedlicher Weise benötigt werden und die auch für die sozialmedizinische Arbeitsfähigkeitsbeschreibung relevant wären.

Die Internationale Klassifikation der Funktionsfähigkeit, Behinderung und Gesundheit ICF [WHO, 2001] ist ein Glossar, in dem potentielle Einflussfaktoren für Gesundheitsprobleme definiert sind. Dies sind einerseits Körperfunktionen und -strukturen, andererseits verschiedene Aspekte von Aktivitäten und Partizipation. Arbeitsbezogen gibt es in der ICF z.B. in einem Kapitel “Arbeit und Beschäftigung“ die Begriffe „Vorbereitung auf Erwerbstätigkeit“, „eine berufliche Tätigkeit erhalten, behalten und beenden“, „bezahlte Tätigkeit“, „unbezahlte Tätigkeit“. Die ICF an sich ist ein Begriffsglossar und darf nicht als Assessment- oder Beurteilungsinstrument missverstanden werden. Der zentrale Nutzen der ICF ist jedoch ihre Grundsatzphilosophie: $\mathrm{Ob}$ eine Beeinträchtigung besteht oder nicht, liegt nicht (nur) an der Erkrankung, sondern an den Aktivitätsanforderungen. Eine Untersuchung zur Qualität von sozialmedizinischen Arbeitsunfähigkeitsbescheinigungen fand im Zeitraum 2007-2009 eine Häufigkeitszunahme von Beschreibungen von "Aktivitätsbeeinträchtigungen“ im Sinne dieser ICF-Philosophie [Linsing et al., 2012]. Auch wurde die ICF als ein Klassifikationsrahmen von Bedingungsfaktoren der Rückkehr an die Arbeit genutzt [Muijzer et al., 2012; Cornelius et al., 2011]. Diese Entwicklungen 
weisen darauf hin, dass die Beschreibung von Beeinträchtigung sich zunehmend in Richtung Aktivitäts- und Fähigkeitsbeschreibung orientiert. Dies liefert auch für die klinischen Praxis hilfreiche Impulse [Nilsing et al., 2011; Glässel et al., 2011].

\section{Aktivitäts- und Fähigkeitsbeeinträchtigungen differenziert beschreiben}

Neben der globalen Beschreibung von Arbeitsfähigkeit gibt es differenziertere Beschreibungen im Sinne von unterschiedlichen arbeitsbezogenen Aktivitäten und Fähigkeiten. Ein solcher Ansatz wird in der Endicott Work Productivity Scale EWPS [Endicott \& Nee, 1997] verfolgt. Die EWPS ist ein Selbstbeurteilungsfragebogen mit 25 Items zur Beschreibung von verschiedenen arbeitsbezogenen Aktivitäten und auch Defiziten in der Arbeitsproduktivität. Der Patient wird gefragt, ob er/sie in den letzten sieben Tagen bei der Arbeit gewesen ist, und falls ja, wie oft eine Reihe von danach aufgelisteten Problemen auftrat. Einige Fragen zielen darauf ab, ob die Person während der Arbeitszeit effektiv gearbeitet hat, d.h. ob sie zu spät auf der Arbeit erschien oder den Arbeitsplatz vor regulärer Feierabendzeit verließ oder während der Arbeitszeit nicht arbeitete aufgrund von Tagträumen, Sorgen oder in die Luft starren oder zu langsam arbeitete oder zu lange benötigte, um Arbeitsergebnisse fertigzustellen. Es wird nach arbeitszeitverschwendendem Verhalten gefragt, z.B. nach Fehlern oder verlegten Arbeitsmaterialien oder Informationen, vergessenen Erledigungen, vergessenen Antworten auf Anfragen. Ein weiterer Abschnitt widmet sich ungünstigen psychischen Zuständen, die bei der Arbeit erlebt wurden, bspw. Einschlafen oder Konzentrationsschwierigkeiten, zu erschöpft zu sein, um zu arbeiten, Ruhelosigkeit am Arbeitsplatz oder Langweile oder Interessenverlust. 
Vermeidungsverhalten wird hinsichtlich sozialer Aspekte erfragt, bspw. Vermeiden von Meetings, Interaktionen mit Kollegen, Vorgesetzten oder Kunden. Schwieriges Interaktionsverhalten wird erfragt hinsichtlich Ungeduld oder gezeigten Ärgeraffekten gegenüber anderen bei der Arbeit. Zuletzt werden auch Arbeitsorganisationsprobleme erfragt, bspw. Schwierigkeiten die eigene Arbeit zu organisieren, Prioritäten zu setzen, oder Arbeiten nicht abzuschließen. Es wurde auch direkt erfragt, ob der Proband seine eigene Produktivität und Effizienz bei der Arbeit als niedriger als erwartet einschätzt. Hinsichtlich erlebter Unterstützungsbedürftigkeit wird erfragt, ob Vorgesetzte oder Kollegen den Probanden an etwas erinnern mussten, oder ob Kollegen eine Arbeit des Probanden noch einmal machen mussten. Das EWPS beschreibt also Arbeitsfähigkeit auf einem Niveau konkreter Aktivitäten, kombiniert mit einigen Aspekten von affektiven und potentiell auch psychopathologischen Zuständen (sich gelangweilt fühlen, rastlos, einschlafen, Konzentrationsprobleme).

Eine rein aktivitäts- und fähigkeitsorientierte Beschreibung von Arbeitsfähigkeit ist mit dem Mini-ICF-APP möglich [Linden et al., 2009]. Das Mini-ICF-APP ist ein Fremdbeurteilungsinstrument zur Beschreibung von Fähigkeits- und Teilhabebeeinträchtigungen aufgrund psychischer Erkrankungen. Es ist international validiert [Linden et al., 2015; Molodynski et al., 2013; Balestrieri et al., 2013] und wird in Gutachterleitlinien als Grundlage für die klinische Arbeitsfähigkeitsbeurteilung im sozialmedizinischen Sinne empfohlen [DRV, 2012; SGPP, 2012]. Das Mini-ICF-APP beschreibt 13 Dimensionen psychologischer Fähigkeiten, die häufig bei psychischen Erkrankungen beeinträchtigt sind: Anpassung an Regeln und Routinen, Planung und Strukturierung von Aufgaben, Flexibilität und Umstellungsfähigkeit, Kompetenz- 
und Wissensanwendung, Entscheidungs- und Urteilsfähigkeit, Widerstands- und Durchhaltefähigkeit, Selbstbehauptungsfähigkeit, Konversation und Kontaktfähigkeit, Gruppenfähigkeit, Selbstpflege und Selbstversorgung, Verkehrsfähigkeit und Mobilität, Proaktivität und Spontanaktivitäten, Fähigkeit zu engen dyadischen Beziehungen. Um das Ausmaß einer Fähigkeitsbeeinträchtigung einzuschätzen, ist ein konkretes Anforderungsprofil notwendig. Für die Beschreibung der Arbeitsfähigkeit bedeutet dies, dass die Fähigkeitsbeeinträchtigung entweder auf den bestehenden Arbeitsplatz (z.B. Krankenschwester im Blutspendedienst), oder auf das Berufsfeld (z.B. Krankenschwester), oder auf einen qualifikationsentsprechenden Arbeitsplatz auf dem allgemeinen Arbeitsmarkt (z.B. soziale, helfende, büro-organisatorische Tätigkeiten) erfolgen muss. Die Beeinträchtigungsschwere kann von geschulten Klinikern eingeschätzt werden, nachdem im halbstrukturierten klinischen Interview Informationen über die Arbeitsanforderungen erfragt worden sind. Es muss außerdem sichergestellt sein, dass es sich um krankheitsbedingte Beeinträchtigungen handelt [Linden et al., 2015]. Für jede Fähigkeitsdimension kann die Beeinträchtigungsschwere eingeschätzt werden. Die Einschätzung ist ein klinisches Expertenurteil. Der Beurteiler soll das Ausmaß der Beeinträchtigung auf Basis seiner Beobachtungen und der explorierten Informationen einzuschätzen. Gibt es eine Einschätzung, dass hinsichtlich einer arbeitsrelevanten Fähigkeit regelmäßig Probleme auffallen (mittelgradige Beeinträchtigung) oder regelmäßig Unterstützung von Dritten notwendig wird (schwere Beeinträchtigung), so ist dies ein Hinweis auf eine mögliche Arbeitsunfähigkeit.

Der Work Limitations Questionnaire WLQ [Lerner et al., 2001] ist ein 
international validierter Selbstbeurteilungsfragebogen zu erlebten Arbeitsfähigkeitsbeeinträchtigungen. Der WLQ wurde bei psychischen Erkrankungen und anderen Erkrankungen verwendet [Lerner et al., 2001] sowie in der Präsentismusforschung [Despiégel et al., 2012]. Im WLQ werden mit 25 Items Arbeitsanforderungen in den Bereichen zwischenmenschliche Anforderungen, Zeitmanagement, Ergebnisse und physische Anforderungen beschrieben. Die Probanden werden gebeten, ihre gesundheitsbezogenen Fähigkeiten bzw. Beeinträchtigungen in der Erfüllung bestimmter Arbeitsanforderungen in den letzten zwei Wochen einzuschätzen. Zunächst werden die Probanden gebeten, die Menge an Zeit anzugeben, während der ihre körperlichen oder psychischen Probleme es ihnen schwer machten verschiedene Arbeitsaktivitäten auszuführen wie bspw. Dinge am Stück und ohne Pause zu erledigen oder sich an Regeln und vorgegebene Zeitpläne zu halten oder seine Aufmerksamkeit auf die Arbeit fokussiert zu halten oder mit anderen telefonisch, live zu sprechen oder die Arbeitslast zu bewältigen. Zusätzlich wird gefragt, wie viel Arbeitszeit die Person in der Lage war, verschiedene Aktivitäten ohne gesundheitsbezogene Probleme auszuführen: sich zu und an verschiedenen Arbeitsorten zu bewegen, Equipment und Technik am Arbeitsplatz zu nutzen. Es geht im WLQ explizit um gesundheitsbedingte Arbeitsprobleme, was einem sozialmedizinischen Verständnis von Arbeitsfähigkeit nahe kommt.

\section{Anwendungsmöglichkeiten}

Die skizzierten Beispiele zeigen die Breite des wissenschaftlichen Verständnisses von Arbeitsfähigkeit und die Notwendigkeit einer begründeten Auswahl von 
Beschreibungsinstrumenten für verschiedene Zwecke.

Es gibt Beschreibungsansätze von Arbeitsfähigkeit

- auf sehr globalen Ebenen einerseits (z.B. 1-Item-Scores: IMET), oder andererseits sehr spezifischer Aktivitäten (z.B. EWPS, WLQ),

- auf der Basis von Arbeitsanforderungen als Bezugspunkt (z.B. Mini-ICFAPP), oder Bezug nehmend auf die frühere beste Arbeitsfähigkeit (z.B. ein Item im Work Ability Index), oder ohne Bezugnahme auf einen bestimmten Arbeitskontext (z.B. ICF-Items).

- ohne [Vinokur et al., 2011] oder mit (WAI, Mini-ICF-APP, IMET) der Annahme zugrundeliegender Krankheitssymptome bzw.

Gesundheitsprobleme

- mit Nutzung kontinuierlicher Likert-Skalen (z.B. EWPS) für die Beschreibung des Beeinträchtigungsausmaßes oder definierter Beeinträchtigungsstufen (z.B. Mini-ICF-APP, GAF Score).

Es stellt sich die Frage, welche Maße für welche praktischen Anwendungszwecke verwendbar sein können.

\section{Sozialmedizinische Beurteilung}

Die sozialmedizinische Beurteilung und Beschreibung der Arbeitsfähigkeit eines Patienten ist immer eine klinische Einzelfallentscheidung. Sozialmedizinische Arbeitsfähigkeits-/Erwerbsfähigkeitsbeurteilung basiert auf einer genauen Kenntnis der aktuellen Arbeitsanforderungen bzw. der Arbeitssituation des Patienten (z.B. Buchhalter), der Krankheitssymptomatik und des -verlaufs (z.B. rezidivierende depressive Erkrankung) sowie den daraus sich ergebenden Fähigkeitsbeeinträchtigungen (z.B. rezidivierend beeinträchtigte 
Durchhaltefähigkeit). Die Fähigkeitsbeeinträchtigung führt erst unter bestimmten Arbeitsbedingungen (z.B. Zeitdruck bei Rechnungsschluss am Jahresende) zu Negativ-Konsequenzen (z.B. Fehler, Verzögerungen) [Muschalla und Linden, 2011]. Arbeitsunfähigkeit im sozialmedizinischen Sinne liegt vor, wenn durch die krankheitsbedingten Fähigkeitsbeeinträchtigungen die Arbeitsausübung soweit beeinträchtigt ist, dass sie ohne Hilfe nicht mehr machbar ist. Arbeitsunfähigkeit läge auch vor, wenn eine Fortsetzung der Arbeitstätigkeit prognostisch eine Verschlimmerung der Erkrankung mit sich bringen würde [AU-Richtlinien, GBA, 2014]. Die Arbeitsfähigkeitsbeurteilung ist immer eine Einzelfallentscheidung, und es kann hierfür keine Normwerte aus Bevölkerungsstichproben geben. Die Arbeitsfähigkeit kann auch nicht auf Grundlage der Selbsteinschätzung des Patienten geschehen, sondern erfordert eine Beobachter-Einschätzung (Expertenurteil). Die sozialmedizinische Arbeitsfähigkeitsbeurteilung wird im ambulanten Bereich von Ärzten vorgenommen. In stationären Settings, wie zum Beispiel der psychosomatischen Rehabilitation, werden sozialmedizinische Arbeitsfähigkeitsbeurteilungen auf Grundlage von Beiträgen verschiedener Therapeuten im Reha-Team und unter Verantwortung eines Arztes formuliert. Arbeitsfähigkeitsbeurteilung kann auf einer einzelnen Exploration beruhen, wie bspw. in einer ambulanten Begutachtungs-Situation. Sie kann aber auch Ergebnis vielfältiger Verhaltensbeobachtungen und ggf. Arbeitsproben im Behandlungsverlauf sein, z.B. in einer stationären Rehabilitation. In beiden Settings kann als Strukturierungshilfe zur Beschreibung der Art und Schwere von arbeitsbezogenen Beeinträchtigungen bei psychischen Erkrankungen das MiniICF-APP [Linden et al., 2009] genutzt werden. Die Inhalte aktivitäts- und fähigkeitsorientierter Fragebögen und Interviewleitfäden können Anregungen für 
die Exploration geben (z.B. EWPS; WHODAS; GSDS; Linden et al., 2015). Im Fall einer Beurteilung der Arbeits- und Erwerbsfähigkeit von Menschen, die arbeitssuchend sind (also einer Beurteilung für den sogenannten ,allgemeinen Arbeitsmarkt"), könnte als Anforderungsmetapher ein „Hotel“" gewählt werden: Die Frage wäre, ob jemand in einem Mittelklassehotel in irgendeiner Position tätig sein könnte. Je nach beruflicher Qualifikationsstufe könnte die Frage sein, ob jemand im Büro in der Buchhaltung oder am Empfang oder als Gärtner oder Handwerker oder in der Küche oder im Management eines Hotels eingesetzt werden könnte.

\section{Psychotherapeutische Behandlung}

Im therapeutischen Kontext können auch über die Frage der Arbeitsfähigkeit hinausgehende Fragen auftreten z. B., ob ein Patient bestimmten Anforderungen im Alltagsleben oder aber in der Behandlung selbst gewachsen ist. Hier könnte es bspw. bei der Beantragung einer Rehabilitationsbehandlung um eine Beschreibung von „Rehafähigkeit“ gehen oder darum festzustellen, ob jemand zu einer Arbeit in einer Werkstatt für Menschen mit Behinderungen in der Lage ist. Hierbei könnte man Mindestanforderungen an eine Behandlungsteilnahme definieren, z.B. was jemand zur Teilnahme an einer Ergotherapie-HandwerkGruppe können muss. Dieses Prinzip entspricht standardisierten situativen Tests [Beispiele in MacDonald et al., 2001].

Den Anforderungs- und Kontextbezug zu berücksichtigen ist auch hinsichtlich der Evaluation beruflicher Rehabilitations-Maßnahmen für Patienten mit chronischen psychischen Erkrankungen und gefährdeter Erwerbsfähigkeit notwendig. Die Kontextanpassung ist hier eine wichtige Behandlungsebene: 
Arbeitsfähigkeit aufgrund von Kontextanpassungen wiederherzustellen bedeutet, dass ein Patient trotz einer bleibenden Erkrankungssymptomatik und bleibender Beeinträchtigungen wieder arbeiten gehen kann: Sein Arbeitsplatz wird an sein reduziertes Leistungsvermögen angepasst. So wird zum Beispiel auch in der neueren Präsentismusforschung der Unterscheidung von Symptom/Krankheit einerseits und Fähigkeit/Aktivität bereits Rechnung getragen. Fragebögen erfassen nicht mehr nur, ob jemand „,bei der Arbeit erscheint, auch wenn es ihm nicht gutgeht“. Stattdessen wird nun gefragt, ob jemand „bei der Arbeit erscheint, auch, wenn es ihm nicht gutgeht, weil er die Arbeit an seinen aktuellen Zustand anpassen kann“ [Thun et al., 2015].

\section{Forschung}

Im Forschungskontext kann es notwendig werden Arbeitsfähigkeit(sbeeinträchtigung) zu quantifizieren, oder Arbeitsfähigkeit in heterogenen Stichproben zu beschreiben, z.B. bei Menschen mit unterschiedlichen Erkrankungen oder aus verschiedenen Berufsgruppen. Wenn es die Fragestellung erfordert, wissenschaftliche Arbeitsfähigkeitsbeschreibungen für viele Menschen im Hinblick auf einheitliche Kriterien zu erhalten, ist eine Standardumwelt bzw. Standardbedingungen zu definieren, auf die sich alle Beurteilungen beziehen sollen. Dies könnten z.B. Mindestanforderungen für eine leichte Tätigkeit auf dem allgemeinen Arbeitsmarkt sein oder Mindestanforderungen im Berufsfeld Krankenschwester.

Wie sind nun wissenschaftliche Untersuchungen, die sich der „Arbeitsfähigkeit““ widmen, für Praktiker zu verstehen und zu beurteilen? Es gilt, dass erst einmal überprüft werden sollte, was eigentlich untersucht worden ist. 
Anhand der Iteminhalte der eingesetzten Instrumente kann man erkennen, ob Arbeitsfähigkeit im Sinne der sozialmedizinischen Definition gemeint ist, oder etwas anders. Es wird erkennbar welche diagnostischen Ebenen (Symptome/Wohlbefinden oder Fähigkeiten/Aktivitäten?) im Mittelpunkt stehen. Definitionen und Zielsetzungen verschiedener Forschungskonzepte zum Thema Arbeitsfähigkeit beurteilen zu können ist insbesondere vor dem Hintergrund wichtig, als sich politische Entwicklungsrichtungen und auch arbeits- und personbezogene Interventionsentwicklungen auf Forschungsergebnisse zur „Arbeitsfähigkeit“" beziehen [van den Berg et al., 2008].

\section{Interessenkonflikte:}

P.A. und C.K. geben an, dass keine Interessenkonflikte bestehen. B.M. ist Mitautorin des Mini-ICF-APP 


\section{Literatur}

Anner J, Schwelger U, Kunz R, Trezzini B, de Boer W: Evaluation of work disability and the international classification of functioning, disability and health: what to expect and what not. BMC Public Health 2012;12:470.

Balestrieri M, Isola M, Bonn R, Tam T, Vio M, Linden M, Maso E: Validation of an Italian version of the Mini-ICF-APP, a short instrument for rating activity and participation restrictions in psychiatric disorders. Epidemiol Psychiatr Sci 2013;22:81-91.

BPtK Bundespsychotherapeutenkammer (2015): BPtK-Studie zur Arbeitsunfähigkeit. Psychische Erkrankungen und Krankengeldmangement. Berlin, BPtK, 2015.

Cornelius LR, van der Klinik JJL, Groothoff JW, Brouwer S: Prognostic Factors of Long Term Disability due to Mental Disorders: A Systematic Review. J Occup Rehabil 2011;21:259-274.

Deck R, Mittag O, Hüppe A, Muche-Borowski C, Raspe: Index zur Messung von Einschränkungen zur Teilhabe (IMET) - Erste Ergebnisse eines ICF-orientierten Assessmentinstruments. Prax Klin Verhaltensmed Rehabil 2007;76:113-120.

Deck R, Walther AL, Staupendahl A, Katalinic A: Einschränkungen der Teilhabe in der Bevölkerung - Normdaten für den IMET aufgrund eines Bevölkerungssurveys in Norddeutschland. Rehabilitation 2015;54:402-410.

DeJong A, Giel R, Sloof C, Wiersma D: Social disability and outcome in schizophrenic patients. Br J Psychiatry 1985;147:621-636.

Despiégel N, Danchenko N, Francois C, Lensberg B, Drummond MF: The use and performance of productivity scales to evaluate presenteeism in mood disorders. Value Health 2012;15:1148-1161.

DRV Deutsche Rentenversicherung Bund. Leitlinien für die sozialmedizinische Begutachtung. Sozialmedizinische Beurteilung bei psychischen und Verhaltensstörungen. Berlin: DRV, 2012.

Endicott J, Nee J: Endicott Work Productivity Scale (EWPS). A new measure to assess treatment effects. Psychopharmacol Bull 1997;33:13-16.

Esposito E, Wang JL, Williams JV, Patten SB. Mood and anxiety disorders, the association with presenteeism in employed members of a general population. Epidemiol Psichiatr Soc 2007;16:231-237.

Foster EE, Pinals DA: Mental impairments as determined in Social Security disability claims. J Am Academy Psychiatr Law 2014;42:379-381.

French JRP Jr: Person role fit. Occup Ment Health 1973;3:15-20.

GBA (2014). Richtlinie des Gemeinsamen Bundesausschusses über die Beurteilung der Arbeitsunfähigkeit und die Maßnahmen zur stufenweise Wiedereingliederung nach $§ 92$ Abs. 
1 und 2 Nr. 7 SGB V (Arbeitsunfähigkeits-Richtlinie). Gemeinsamer Bundesausschuss, Bundesanzeiger AT 27.01.2014 B4 in Kraft getreten am 28. Januar 2014.

Gatchel RJ, Polatin PB, Mayer TG, Garcy PD: Psychopathology and the rehabilitation of patients with chronic low back pain disability. Arch Phys Med Rehabil 1994;75:666-670.

Glässel A, Finger ME, Cieza A, Treitler C, Coenen M, Escorpizo R: Vocational rehabilitation from the client's perspective using the International Classification of Functioning, Disability and Health (ICF) as a reference. J Occup Rehabil 2011;21:167-178.

Ilmarinen J, Lehtinen S (Hrsg): Past, Present and Future of Work Ability - People and Work Research Reports, 65. Finnish Institute of Occupational Health, 2004.

Lederer V, Loisel P, Rivard M, Champagne F: Exploring the diversity of conceptualizations of work (dis)ability: a scoping review of published definitions. J Occup Rehabil 2014;24:242-267.

Lerner D, Amick BC, Rogers WH, Malspeis S, Bungay K, Cynn D: The Work Limitations Questionnaire. Medical Care 2001;39:72-85.

Lidwall U: Sick leave diagnoses and return to work: a Swedish register study. Disabil Rehabil 2015;37:396-410.

Linden M, Baron S, Muschalla B: Mini-ICF-Rating für psychische Störungen (Mini-ICFAPP). Ein Kurzinstrument zur Beurteilung von Fähigkeits- bzw. Kapazitätsstörungen bei psychischen Störungen. Göttingen, Hans Huber, 2009

Linden M, Baron S, Muschalla B, Ostholt-Corsten M: Fähigkeitsbeeinträchtigungen bei psychischen Erkrankungen. Diagnostik, Therapie und sozialmedizinische Beurteilung in Anlehnung an das Mini-ICF-APP. Göttingen, Hogrefe, 2015.

MacDonald-Wilson K, Rogers ES, Anthony WA: Unique issues in assessing work function among individuals with psychiatric disabilities. J Occup Rehabil 2001;11:217-232.

Molodynski A, Linden M, Juckel G, Yeeles K, Anderson C, Vazquez-Montes M, Burns T: The reliability, validity, and applicability of an English language version of the Mini-ICFAPP. Soc Psychiatr Psychiatric Epidemiol 2013;48:1347-1354.

Muijzer A, Brouwer S, Geertzten JH, Groothoff JW: Exploring factors relevant in the assessment of the return-to-work process of employees on long-term sickness absence due to a depressive disorder: a focus group study. BMC Public Health 2012;6:12:103.

Muschalla B: Fähigkeitsorientierte Verhaltenstherapie bei psychischen Erkrankungen. Verhaltenstherapie 2014;24:48-55.

Muschalla B, Fay D, Jöbges M, Linden M, Ayhan H, Flöge B, Heidrich ML: Evaluation einer Gruppentherapie für arbeitsplatzbezogene Ängste und Arbeitsplatzphobie. 
Abschlussbericht und Behandlungsmanual zum DRV-Forschungsprojekt. Brandenburgklinik Bernau und Universität Potsdam, Arbeits- und Organisationspsychologie, 2014.

Muschalla B, Linden M: Sozialmedizinische Aspekte bei psychischen Erkrankungen. Teil 1: Definition, Epidemiologie, Kontextbedingungen und Leistungsbeurteilung. Nervenarzt 2011;82:917-931.

Muschalla, B., Vilain, M., Lawall, C., Lewerenz, M., \& Linden, M. (2012). Participation restrictions at work indicate participation restrictions in other domains of live. Psychology, Health \& Medicine, 17, 95-104.

Nilsing E, Söderberg E, Normelli H, Oberg B: Description of functioning in sickness certificates. Scand J Public Health 2011;39:508-516.

Sass H, Wittchen HU, Zaudig M, Houben I: Diagnostische Kriterien des DSMIV. Göttingen, Hogrefe, Verlag für Psychologie, 1998.

SGPP Schweizerische Gesellschaft für Psychiatrie und Psychotherapie: Qualitätsleitlinien für psychiatrische Gutachten in der Eidgenössischen Invalidenversicherung. Bern, SGPP, SGVP, 2012.

Sheehan DV: The Anxiety Disease, Charles Scribner \& Sons, New York, 1983, page 151.

Stansfeld SA, Clark C, Caldwell T, Canner R, North F, Marmot M: Psychosocial work characteristics and anxiety and depressive disorders in midlife: the effects of prior psychological distress. Occup Environ Med 2008;65:634-642.

Thun S, Rostad I, Saksvik PO: How to measure sickness presenteeism? Poster presentation on the EAWOP congress, May, 2015, Oslo.

Tuomi K, Ilmarinen J, Jahkola A, Katajarinne L, Tulkki A. Work Ability Index, 2nd revised edition. Helsinki, Finnish Institute of Occupational Health, 1998.

Tziner A, Meir EI, Segal H: Occupational Congruence Personal Task-Related Attributes: How Do They Relate To Work Performance? J Career Assessment 2002;10:401-412.

Van den Berg TIJ, Elders LAM, de Zwart BCH, Burdorf A: The effects of work-related and individual factors on the Work Ability Index: a systematic review. Occup Environ Med 2008;66:211-220.

Vinokur AD, Pierce PF, Lewandowski-Romps L, Hobfoll SE, Galea S: Effects of war exposure on air force personnel's mental health, job burnout and other organizational related outcomes. J Occup Health Psychol 2011;16:3-17.

WHO World Health Organization: International Classification of Functioning, Disability and Health (ICF). Geneva, World Health Organization, 2001.

WHO World Health Organization: WHODAS II - Disability Assessment Schedule Training Manual: A guide to administration. Geneva, World Health Organization, 2004. 
Wiersma D, DeJong A, Ormel J: The Groningen Social Disability Schedule:

Development, relationship with the I.C.I.D.H., and psychometric properties. Int J Rehabil Res 1988;11:213-224.

Wiersma D, DeJong A, Kraaijkamp HJM, Ormel J: The Groningen Social Disabilities Schedule. Manual and Questionnaires, 2nd Version. University of Groningen, Department of Social Psychiatry, WHO, 1990. 
